\title{
Innovations in and the Changing Landscape of Geography Education with Geographic Information Systems
}

Patrick Rickles and Claire Ellul

Department of Civil, Environmental and Geomatic Engineering, University College London, London, United Kingdom

Patrick Rickles

UCL Chorley Institute

University College London

Pearson Building

Gower Street

London

WC1E 6BT

United Kingdom

Phone: +44 (0)20 76792452

Email: p.rickles@ucl.ac.uk
Dr. Claire Ellul

Department of Civil, Environmental and Geomatic Engineering

Chadwick Building

Gower Street

London

WC1E 6BT

United Kingdom

Phone: +44 (0)20 76794118

Email: c.ellul@ucl.ac.uk 


\section{Innovations in and the Changing Landscape of Geography Education with Geographic Information Systems}

\section{Opportunities and challenges for educators with Geographic Information}

\section{Systems}

As we progress through the 21 st century, humanity continues to face an array of complex global challenges that require perspectives from many knowledge areas to understand and cooperation across them to devise solutions. Tackling some of the issues associated with climate change, for example, will involve collaboration between environmental scientists, biologists, sociologists and many others, as these do not affect a solitary discipline. The technologies being used by these researchers, and continued advances in them, are opening up new analytical opportunities; however, in order to prepare people to use these technologies, they will need to learn how to use them. These technologies may not yet be available for formal education at schools or universities, though, and the education sector overall is known to lag behind others in regards to integrating technologies (Morrison \& Lowther, 2002). Educators must therefore adapt their practices in creative ways to integrate tools as possible in order to ensure students learn what is necessary.

Technologies that collect locational, real-world data, directly or indirectly, can be extremely useful in a variety of projects. As everything that happens happens somewhere in space and time (Anselin, Florax \& Rey, 2013), recording and understanding locational aspects of data can be vital for informed decision-making. The incorporation of geographical analyses to understand location provides a crossdisciplinary medium for communication and understanding; one of the most powerful tools for these types of analyses, said to be a fundamental research tool itself (Chen, 1998), is a Geographic Information System (GIS). A GIS is defined as a system with the 
ability to capture, store, analyse, manage and present data that are linked to geographical locations (Bhat, Shah \& Ahmad, 2011); however, such systems are also difficult to use due to hardware issues, a lack of training and incompetence using the software (Liu, Tan \& Xiang, 2012). It is therefore important for educators to not only teach GIS concepts of relevance to their audience of learners, but also any peripheral topics that may be necessary as well.

Previously largely a specialist tool, day-to-day use of GIS has become more common, largely due to smartphones and geospatially enabled apps. It was estimated that by 2017, 2.6 billion people globally, over a third of the world's total population, would own smartphones (Statista, 2016). It was also reported at Apple's Worldwide Developers Conference in 2015 that Apple Maps received 5 billion requests per week (Elmer-DeWitt, 2015), which does not include requests made to other similar apps (e.g. Google Maps). The combination of these factors means that there is a phenomenally large number of non-expert users of GIS and, therefore, a variety of opportunities for GIS in commercial and research projects. These potentials are attracting new GIS learners who have their own ideas for GIS applications and care coming from different knowledge areas (e.g. anthropology, political science, etc.) and backgrounds (e.g. adult learners seeking continued professional development). While education and learning in GIS is lifelong, it often starts in the more formal context of the classroom. Educators must therefore keep up to date with emerging technological trends, and incorporate leading-edge research in their teaching, while working in a discipline where technology and trends rapidly change. Nevertheless, there is a growing community of GIS educators, such as the contributors to this symposium, who are using their experience and inventiveness to address these issues and reach new audiences. 


\section{The papers}

GIS educators have come together to share their successes and try to devise ways of overcoming obstacles through a series of sponsored sessions that have been hosted for five consecutive years at the Royal Geographical Society’s annual international conference on interdisciplinary GIS applications and educational initiatives ${ }^{12345}$. These were met with enthusiasm from presenters and attendees alike, and brought together people to share their successes and lessons to help improve and expand the application of GIS. A previous symposium, arranged by the convenors of those sessions, shared some of the presented interdisciplinary GIS applications (Ellul, 2015); the papers in this new symposium focus on how educators have attempted to align and adjust their practices as well as learning objectives to meet an increasingly diverse student population's needs over time. The themes that have emerged from them are as follows:

${ }^{1}$ Rickles, P. (Chair) \& Ellul, C. (2012, July). Geography in Interdisciplinary Research: Threat or Opportunity?. Session conducted at the Royal Geographical Society with IBG Annual International Conference, Edinburgh, UK.

${ }^{2}$ Rickles, P. (Chair) \& Ellul, C. (2013, August). Using GIS in Interdisciplinary Research: Natural and Social Sciences Making the Case. Session conducted at the Royal Geographical Society with IBG Annual International Conference, London, UK.

${ }^{3}$ Rickles, P. (Chair) \& Ellul, C. (2014, August). GIS Education: Who Wins? Session conducted at the Royal Geographical Society with IBG Annual International Conference, London, UK.

${ }^{4}$ Rickles, P. \& Ellul, C. (Chair) (2015, September). GIS and the Anthropocene: Educational Perspectives. Session conducted at the Royal Geographical Society with IBG Annual International Conference, Exeter, UK.

${ }^{5}$ Rickles, P. \& Ellul, C. (Chair) (2016, August). Learning GIS: Establishing the Nexus Between Disciplines. Session conducted at the Royal Geographical Society with IBG Annual International Conference, London, UK. 
- Evolution of Formal Education: how GIS programmes have evolved and continue to do so to accommodate new learners

- Online Education: reaching learners who may not be able to access resources, either due to locational or time issues

- Adult Education: adapting educational techniques to ensure relevance of learnings for immediate and specialised applications

- Incorporation of Online and Mobile Technology: integrating the latest geospatial technologies in teaching materials, while bearing in mind existing institutional infrastructure

The first two papers (Nigel Walford; Nick Tate and Claire Jarvis) focus on the theory behind educational practices for GIS programmes, the following two (Tom Argles; Joana Barros) discuss the use of online education for GIS and the final one (Britta Ricker and Jim Thatcher) combines online and mobile platforms to introduce innovative approaches in GIS education.

Nigel Walford chronicles the progression of formal GIS programmes over time at higher education institutions in the UK and their adaptation to meet research and disciplinary needs. At the postgraduate as well as undergraduate levels, it is necessary to balance the introduction and incorporation of emerging technologies while at the same time maintaining conceptual integrity. This was - and continues to be - a requirement in order to train people to use GIS appropriately for the work that they are to undertake as part of their professional practices. GIS has also begun to be offered as a course at an introductory level in Geography programmes as well as in those of other disciplines, providing a sample of what is possible with GIS; previously these were largely meant for those training to become specialists in GIS. This diffusion amongst disciplines, 
along with exposure to "geographically enabled" devices, has helped to widen the potential user base of GIS. It is also noted that higher as well as further education programmes using GIS face issues in the differences between theory and practice and the need to accommodate different learners with different goals - a nuance that requires further investigation.

Nick Tate and Claire Jarvis suggest the establishment of a community of practice to support collaborative learning efforts for those interested in learning GIS. A community of practice may be particularly complimentary to the social nature of contemporary applications of GIS, given its ubiquity in professional and non-professional settings across knowledge domains. Expanded access to GIS technologies as well as formal and informal educational materials, such as blogs, wikis, podcasts and massive open online courses (MOOCs), has allowed those who are interested to learn in their own time, which may be more conducive to them. These resources also take into account the social aspects of meaningful, situated learning as well as the differences in how people learn. By fostering an inclusive environment, it may be possible to bring together people - online or otherwise - so they may share successes and applications with GIS to inspire, as well as vent their frustrations and discuss how to overcome issues.

Tom Argles discusses the development and evolution of an entirely online course that utilises GIS as a tool for scientific investigation. The compilation of a suite of distance learning GIS resources, and analysis of their usage and uptake, has led to a better understanding of how to support these online learners in understanding GIS concepts and not just simply a mastery of the software. Learning GIS, however, can be difficult; therefore, the aims of this course may have been considered quite ambitious. Nevertheless, some of the outcomes show that forum discussions provided a valuable medium for peer learning and that static and dynamic support mechanisms were overall 
positively received.

Joana Barros explores teaching and learning with GIS at the undergraduate and graduate level at an institution that specialises in part-time, evening-taught, lessons for mature students. These adult learners not only have a clearer idea of learning objectives they wish to achieve, but they also come from diverse professional backgrounds and have varied skillsets, which poses a number of challenges for educators to overcome. Solutions implemented include the incorporation of a standardised GIS curriculum, adjustments to face-to-face and distance learning activities over time and the creation of minimum assessment criteria, allowing those who are interested to go beyond the set requirements. This work recognises that importance of the separate - though interrelated - module design elements of content-related design and settings design in the construction a successful learning experience.

Britta Ricker and Jim Thatcher investigate the educational opportunities created with using mobile technologies to collect and analyse geographical information. This not only opens up the use of GIS to wider audiences but also places greater demands on GIS professionals to be able to support these new user bases; therefore, a flexible pedagogical framework is suggested that teaches GIS students not only GIS and associated concepts, but also about cyber-infrastructures and the analysis of ever growing and diversifying datasets. To create an inclusive educational practice, the framework is organised into levels based upon a learner's knowledge background and suggests how to appropriately construct teaching materials. Through trials with students, transitioning between levels was shown to be challenging; however, learners should be aware that, regardless of level, they could still contribute to and engage with GIS in a variety of ways. 


\section{GIS Education for the Future}

Altogether, the papers of this symposium show the adaptability of educators with years of experience and their techniques in overcoming issues to try to provide students with the best possible environment when learning GIS. These educators have had to design materials to take into consideration cultural and individual learning differences as well as varying levels in digital proficiency. Web and mobile platforms provide new opportunities for teaching and learning, so understandings and acceptance of where learning may come from will need to expand. Given the increasingly prospects for GIS use by new audiences, GIS may begin to be embedded into courses in other disciplines. With that said, though, it is important that constructed materials and resources are based on meaningful, real-world examples that are relevant to the students of that discipline to demonstrate what is possible in their discipline with GIS. Improving the learning journey of GIS to reach new audiences may inspire novel applications for GIS, leading to new ways of understanding and viewing the world. As use of GIS is likely to continue to grow, it benefits educators to encourage all types of learners in achieving their goals, as their developed practices for support will only help to enrich the GIS community.

\section{References}

Anselin, L., Florax, R., \& Rey, S. J. (Eds.). (2013). Advances in spatial econometrics: methodology, tools and applications. Springer Science \& Business Media.

Bhat, M. A., Shah, R. M., \& Ahmad, B. (2011). Cloud Computing: A solution to Geographical Information Systems (GIS). International Journal on Computer Science and Engineering, 3(2), 594-600.

Chen, X.M. (1998). Integrating GIS education with training: A project-oriented approach. Journal of Geography, 97(6), p. 261-268.

Ellul, C. (2015). Geography and Geographical Information Science: Interdisciplinary Integrators. Journal of Geography in Higher Education, 29(2), 191-194. 
Elmer-DeWitt, P. (2015). Why 3.5 times more Apple users choose Apple Maps over Google Maps. Retrieved June 06, 2016 from http://fortune.com/2015/06/16/apple-google-maps-ios/

Liu, Y., Tan, G. C. I., \& Xiang, X. (2012). Singapore: The information technology masterplan and the expansion of GIS for geography education. In A. J. Milson, A. Demirci, \& J. J. Kerski (Eds.), International perspectives on teaching and learning with GIS in secondary schools, (pp. 215-224). Dordrecht: Springer. Morrison, G.R., \& Lowther, D.L. (2002). Integrating computer technology into the classroom. Columbus, $\mathrm{OH}$ : Sage.

Statista. (2016). Smartphones - Statistics \& Facts. Retrieved June 06, 2016 from http://www.statista.com/topics/840/smartphones/ 